\title{
Lagophthalmos in a multibacillary population under multidrug therapy in the People's Republic of China
}

\author{
PAUL COURTRIGHT*, SUSAN LEWALLEN $\dagger$, \\ HUAN-YING LI $\ddagger$, LU-FANG HU§ \& \\ JIAN-WEN YANG§ \\ * Department of Ophthalmology, University of British Columbia, St \\ Paul's Hospital, 1081 Burrand Street, Vancouver, BC, V6Z 1 Y6 \\ Canada; $† 3720$ Harrison Street, Bellingham, WA 98226 USA; \\ $\ddagger$ Beijing Institute of Tropical Medicine, Beijing, Peoples Republic \\ of China; §Sichuan Institute of Dermatology, Chengdu, Sichuan, \\ Peoples Republic of China
}

Accepted for publication 23 March 1995

\begin{abstract}
Summary Lagophthalmos may be the most common potentially blinding ocular condition in leprosy. The magnitude of the problem among multibacillary patients has not been determined. We sought to ascertain the magnitude of lagophthalmos in a multibacillary leprosy patient population under multidrug therapy (MDT) (both newly diagnosed and with a prior history of dapsone monotherapy) in China and assess factors associated with its presence. In a survey of 640 multibacillary patients $3.8 \%$ of the newly diagnosed patients and $10 \cdot 2 \%$ of the patients with prior dapsone monotherapy had lagophthalmos. Corneal disease and vision loss were common in both groups. Poor compliance with MDT, duration between onset and diagnosis, and duration on dapsone monotherapy were associated with the presence of lagophthalmos.

Our findings suggest that there may be a threshold at which MDT must be maintained to prevent lagophthalmos. Early leprosy diagnosis and treatment would also lessen the incidence of lagophthalmos in these patients. The high proportion of lagophthalmos patients with corneal disease suggests that there has been inadequate eye care for these patients.
\end{abstract}

\section{Introduction}

Lagophthalmos may be the most common potentially blinding ocular condition in leprosy. There are few studies with sufficient sampling design to generate estimates of life-time incidence of lagophthalmos in either the pre-multidrug therapy (MDT) era or MDT era. ${ }^{1}$ In the pre-MDT era results from surveys (with appropriate sampling) in Malawi $i^{2}$ and Korea ${ }^{3}$ demonstrated a prevalence of lagophthalmos of $3 \cdot 1 \%$ and $22 \%$, respectively. The Malawian population was young, primarily paucibacillary, and had a 
short duration of disease while the Korean population studied was older, primarily multibacillary, and had a long duration of disease. Brandt and colleagues suggested that antileprosy therapy (dapsone monotherapy) could reduce the incidence of lagophthal$\operatorname{mos}^{4}$ although the contribution of disease type, duration of disease, and duration of chemotherapy was not determined.

Evidence from MDT treated patients in India $^{5}$ suggests that at least $2 \%$ of paucibacillary patients will develop lagophthalmos within 12 months of the onset of disease. The presence of a facial patch may be responsible for at least $85 \%$ of cases. Although lagophthalmos was not recorded in any of the paucibacillary patients in a recent survey of newly diagnosed patients in $\operatorname{Nepal}^{6}$ the study sample was small $(n=107)$.

The magnitude of the problem of lagophthalmos among multibacillary patients is less clear. Prior to the introduction of MDT, evidence from Korea would suggest that the life-time incidence of lagophthalmos in multibacillary patients was at least $25 \%$. In a survey of 1984 multibacillary patients (primarily 'cured' patients) in Jiangsu Province, China, $31 \%$ had lagophthalmos. ${ }^{7}$ In the Nepal survey ${ }^{6} 6 \%$ of multibacillary patients already had lagophthalmos at the time of their disease diagnosis.

We sought to determine the prevalence of lagophthalmos in a multibacillary patient population under MDT and to investigate the factors associated with its presence.

\section{Materials and Methods}

The study was carried out in Liangshan Yi Autonomous Region of Sichuan Province, People's Republic of China. Study methods have been described previously. ${ }^{8}$ Briefly, this involved an assessment of leprosy-related eye conditions by leprosy paramedical workers trained to recognize ocular conditions using a previously tested criteria. ${ }^{9}$ Lagophthalmos was defined as either mild (ability to close with forced pressure but not gentle, i.e. as in sleep) or moderate to severe (inability to close with forced pressure). For the purpose of this report these two groups have been combined. All 'active' leprosy patients in the region (except two remote counties) were examined. Active patients are defined as either patients newly diagnosed (since 1988 in all counties) and treated by MDT, or patients diagnosed as having leprosy prior to 1988 and on dapsone monotherapy until MDT was introduced in their county. Some active patients have completed the two-year MDT regimen and are under surveillance only. Clinical records of a systematic sample (every other patient) were extracted; clinical characteristics collected are given in Table 1. Compliance with MDT was considered good if 24 months of antileprosy therapy was completed within 36 months without any interruption of 3 months or more. As clinical charts were reviewed in a retrospective fashion, facial lesions were defined as either a lesion conclusively involving the lid or a lesion on the face: lid involvement unknown. Cured leprosy patients (released from treatment), which comprise $75 \%$ of the leprosy population in the region, were not included because of the lack of reliable clinical data.

For univariate data analysis Student's $t$-test values (separate variance estimates for those with unequal standard deviations) were calculated for continuous predictor variables and $\chi$-square values (corrected for small sample sizes) for dichotomous variables. For multivariate analysis, Cox's proportional hazards model was used to generate a prevalence rate ratio adjusted for confounding. 
Table 1. Characterstics included in study

\author{
Age \\ Gender \\ Ethnic group \\ Age at diagnosis \\ Kilometres to health centre or health worker \\ Bacteriologic index at diagnosis \\ Bacteriologic index at completion of multidrug therapy \\ Duration between onset and diagnosis \\ Deformity index at diagnosis \\ Deformity index at present \\ Deformity index for hands \& feet at present \\ Facial lesions on face \\ Duration on dapsone monotherapy prior to MDT \\ (only on patients with a history of dapsone monotherapy) \\ Compliance with MDT \\ Type 1 reactions during or after treatment \\ Type 2 reactions during or after treatment \\ History of previous dapsone monotherapy
}

\title{
Results
}

There were 640 multibacillary patients in the study; $208(32.5 \%)$ were newly diagnosed (treated with MDT only) and $432(67 \cdot 5 \%)$ had a history of prior dapsone monotherapy. Good compliance with MDT was recorded in $97 \%$ and the median duration between disease onset and diagnosis in newly diagnosed patients was 24 months. Lagophthalmos was present in 44 patients $(10 \cdot 2 \%)$ with a history of prior dapsone monotherapy and in 8 patients $(3.8 \%)$ who were newly diagnosed. Because of the hazards associated with the pooling of data from these two groups ${ }^{8}$ we describe them separately.

\section{NEWLY-DIAGNOSED LEPROSY PATIENTS}

Lagophthalmos accounted for $35 \%$ of leprosy-related potentially blinding eye disease (defined as lagophthalmos, diminished corneal sensation, acute uveitis, or chronic uveitis) in these patients. Corneal disease (keratitis, ulcer, or opacity) was recognized in $87.5 \%$ of the patients with lagophthalmos compared to $2 \cdot 0 \%$ of the patients without lagophthalmos, Table 2 . Only 4 patients $(1.9 \%)$ had a best-corrected vision (in the better eye) of $<6 / 18$; in three cases this was probably due to cataract. In the patients with lagophthalmos, 5 eyes $(31 \cdot 3 \%)$ had vision $<6 / 18$. In the patients without lagophthalmos only 6 eyes $(1 \cdot 5 \%)$ had a vision $<6 / 18$.

Two of the four patients in whom poor compliance with MDT was reported had lagophthalmos. In patients in which good compliance was recorded the prevalence of lagophthalmos was $3.0 \%$ (Fisher's exact test $p$ value $=0 \cdot 007$ ). Lagophthalmos was slightly more frequent in patients in whom facial lesions were recorded than in patients without facial lesions. Patients with lagophthalmos had a lower bacteriologic index at start of MDT $($ mean $=2.2$, standard deviation $=1 \cdot 1)$ compared to patients without lagophthalmos $($ mean $=3 \cdot 0$, standard deviation $=1 \cdot 1)$. (Student's $t$ test $p=0 \cdot 06)$. 
Table 2. Characteristics associated with the presence of lagophthalmos.

Newly diagnosed leprosy patients

\begin{tabular}{llr}
\hline & \multicolumn{2}{c}{ Lagophthalmos } \\
\cline { 2 - 3 } & $\begin{array}{c}\text { Present } \\
\text { No. }(\%)\end{array}$ & $\begin{array}{c}\text { Absent } \\
\text { No. }(\%)\end{array}$ \\
\hline Visual acuity (better eye) & & \\
$\quad<6 / 18$ & $1(12 \cdot 5)$ & $3(1 \cdot 5)$ \\
$\geqslant 6 / 18$ & $7(87 \cdot 5)$ & $197(98 \cdot 5)$ \\
Corneal disease & & \\
Present & $7(87 \cdot 5)$ & $4(2 \cdot 0)^{*}$ \\
$\quad$ Absent & $1(12 \cdot 5)$ & $196(98 \cdot 0)$ \\
Other leprosy-related def ormities & & \\
$\quad$ Present & $5(62 \cdot 5)$ & $82(41 \cdot 0)$ \\
$\quad$ Absent & $3(37 \cdot 5)$ & $118(59 \cdot 0)$ \\
Facial involvement & & \\
$\quad$ Lid involvement noted & $4(50 \cdot 0)$ & $93(46 \cdot 5)$ \\
$\quad$ Lid involvement possible & $3(37 \cdot 5)$ & $50(25 \cdot 0)$ \\
$\quad$ No facial involvement & $1(12 \cdot 5)$ & $57(28 \cdot 5)$ \\
\hline
\end{tabular}

* Fishers exact (two-tailed) $p$ value $<0.001$.

\section{PATIENTS WITH A PRIOR HISTORY OF DAPSONE MONOTHERAPY}

Lagophthalmos accounted for $46.8 \%$ of the eye disease in these patients. Corneal disease was recognized in $68 \cdot 2 \%$ of patients with lagophthalmos and in $3 \cdot 1 \%$ of patients without lagophthalmos; Table 3 . Poor vision $(<6 / 18$ in the better eye) was recorded in $4 \cdot 2 \%$ of this group. Cataracts probably accounted for $44.4 \%$ of the poor vision in patients with a

Table 3. Characteristics associated with the presence of lagophthalmos. Patients with a prior history of dapsone monotherapy

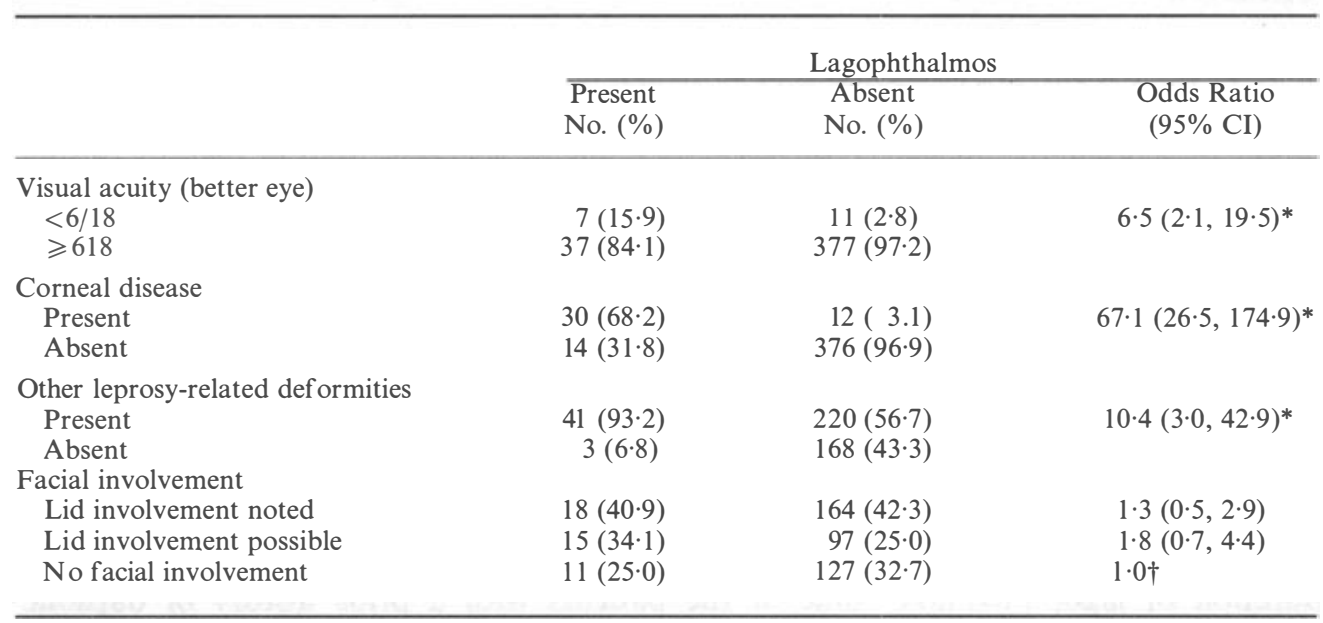

* Chi-square (with Yates correction) $p$ value $<0 \cdot 001$.

$\dagger$ Ref erence group.

CI, Confidence interval. 
Table 4. Selected characteristics associated with the presence of lagophthalmos in patients with a prior history of dapsone monotherapy

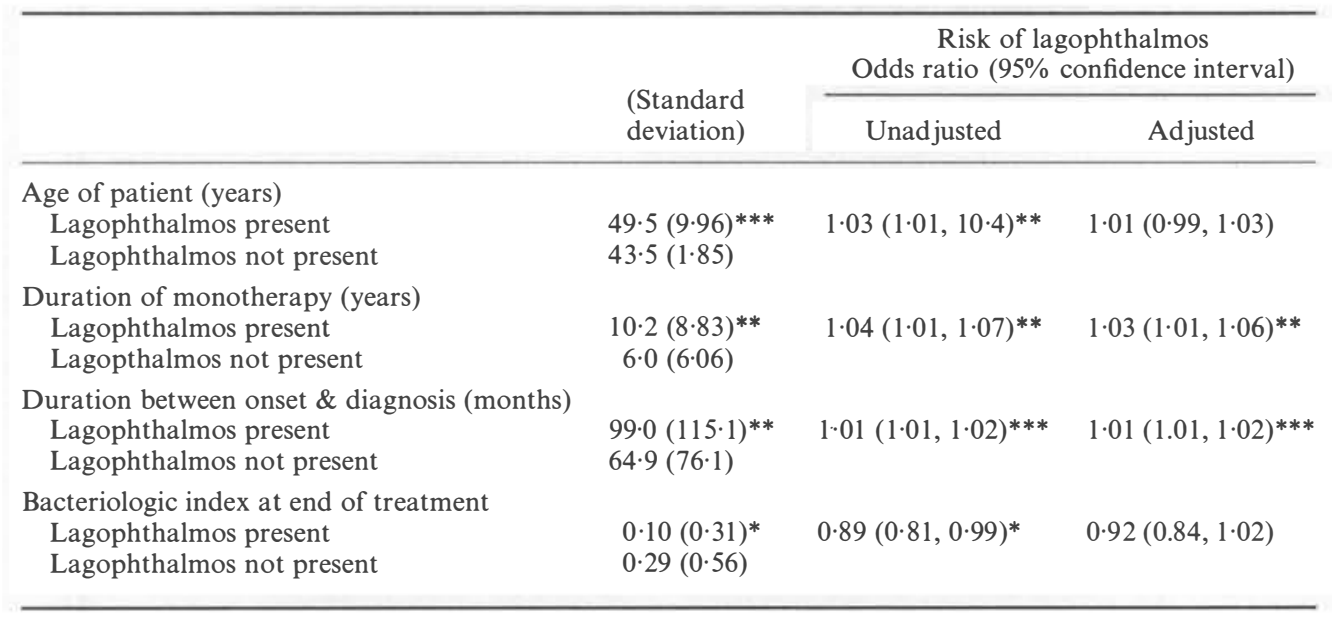

Statistical significance measured at: ${ }^{*} p<0.05,{ }^{* *} p<0.01,{ }^{* * *} p<0.001$.

prior history of dapsone monotherapy. In the patients with lagophthalmos 17 eyes $(19 \cdot 3 \%)$ had a vision of $<6 / 18$ while in the patients without lagophthalmos only 19 eyes $(2 \cdot 4 \%)$ had a vision of $<6 / 18$. Factors associated with the presence of lagophthalmos are shown in Table 4. Multivariate analysis demonstrated that only duration on monotherapy and duration between onset of disease and diagnosis were independently associated with the presence of lagophthalmos.

\section{Discussion}

With good case-finding and good case control it is encouraging that lagophthalmos was recognized in only $3.8 \%$ of the newly diagnosed multibacillary patients. This does not reflect the cumulative incidence of lagophthalmos as adequate steroid therapy during reactions may have resulted in a resolution of lagophthalmos in some cases. ${ }^{10}$ Furthermore, there is a possibility that some patients will develop lagophthalmos after the completion of MDT. The small number of newly diagnosed patients with lagophthalmos in our study limits our ability to investigate characteristics that contribute to lagophthalmos in these patients. Nevertheless, the fact that lagophthalmos was found in two of the four patients in which poor compliance was recorded suggests that there may be a threshold at which MDT must be maintained to prevent lagophthalmos from developing.

Comparing the patients with a prior history of dapsone monotherapy to newly diagnosed patients provides some additional information. We cannot evaluate the duration of lagophthalmos; some of the patients with a prior history of dapsone monotherapy probably had lagophthalmos on diagnosis of leprosy as the duration between onset and diagnosis in these patients was 82 months, considerably longer than the newly diagnosed patients. Our data suggest that some of the bacteriocidal qualities of 
MDT, not found in (bacteriostatic) dapsone, reduce the propensity for damage to the orbicularis oculi. Interestingly, duration of dapsone monotherapy (evidence of insufficient antileprosy therapy) and duration between onset and diagnosis (evidence of prolonged periods in which Mycobacterium leprae proliferate) are independent contributors to the presence of lagophthalmos in patients with a prior history of dapsone monotherapy.

The low bacteriologic index found in patients with lagophthalmos may reflect an increased cellular response by these patients. The independent contribution of facial involvement, reversal reactions, and cellular immunity in multibacillary patients cannot be sufficiently evaluated in our study population.

It was disturbing to find that even among newly diagnosed patients a large proportion of the lagophthalmos patients had corneal disease and that vision loss was common. At the time of the survey, surgical correction of lagophthalmos was not available to leprosy patients in Liangshan. While we do not have the knowledge and skills to prevent lagophthalmos from developing (other than early and adequate treatment) corneal disease and vision loss may be prevented by adequate surgical correction of lagophthalmos. Lagophthalmos need not be a significant contributor to corneal disease and blindness in leprosy.

\section{Acknowledgments}

The China Leprosy Eye Care Project has been supported by Project ORBIS and the Ministry of Public Health, China. Their assistance is greatly appreciated.

\section{References}

${ }^{1}$ Courtright P. Defining the magnitude of ocular complications from leprosy: problems of methodology. Int $J$ Lepr, 1988; 56: 566-573.

2 Ticho U and Ben Sira I. Ocular leprosy in Malawi. Br J Ophthalmol, 1970; 54: 107-112.

${ }^{3}$ Courtright P, Green R, Pilarski R, Smucny J. A survey of the eye complications of leprosy in South Korea. Lepr Rev, 1984; 55: 229-237.

4 Brandt F, Kalthoff PG. The incidence of lagophthalmos and posterior synechiae of the iris during chemotherapy of leprosy (morbus Hansen). Tropenmed Parasit, 1983; 34: 75-78.

${ }^{5}$ Hogeweg M, Kiran KU, Suneetha S. The significance of facial patches and Type 1 reaction for the development of facial nerve damage in leprosy. A retrospective study among 1226 paucibacillary leprosy patients. Lepr Rev, 1991; 62: 143-149.

6 Lubbers WJ, Schipper A, Hogeweg M, de Soldenhoff R. Eye disease in newly diagnosed leprosy patients in eastern Nepal. Lepr Rev, 1994; 65: 231-238.

7 Yan LB, Chang GC, Li WZ, Ye GY, Zheng ZJ, Yant ZM, et al. Analysis for 2114 cases of lagophthalmos in leprosy. China Lepr J, 1993; 9: 6-8.

8 Courtright P, Hu LF, Li HY, Lewallen S. Multidrug therapy and eye disease in leprosy: a cross-sectional study in the People's Republic of China. Int J Epidemiol, 1994; 23: 835-842.

9 Courtright P, Lee HS, Lewallen S. Training for primary eye care in leprosy. Bull WHO, 1990; 68: 347-351.

10 Kiran KU, Hogeweg M, Suneetha S. Treatment of recent facial nerve damage with lagopthalmos, using a semistandardized steroid regimen. Lepr Rev, 1991; 62: 150-154. 